\title{
On the Relation between Boolean Curve Fitting and the Inverse Problem of Boolean Equations
}

\author{
Ali M. Rushdi and Ahmed S. Balamesh \\ Department of Electrical and Computer Engineering, Faculty of Engineering, King Abdulaziz \\ University, P. O. Box 80204, Jeddah 21589, Saudi Arabia \\ arushdi@kau.edu.sa
}

\begin{abstract}
This paper explores the similarities and differences between two prominent problems in the mathematics of Boolean functions. The first of these problems is that of Boolean curve fitting (BCF), also known as Boolean interpolation, which deals with constructing a curve $z=f(\mathbf{X})$ through a number of points $\mathrm{z}_{k}=f\left(\mathbf{X}_{k}\right)$ where $k=1,2, \ldots, m$. The second problem is the Inverse Problem of Boolean equations (IPBE), which constructs a Boolean function whose zeroes are all known. While the problem of Boolean curve fitting might require a consistency condition for its solution, the Inverse Problem of Boolean equations might use a consistency condition as an input. Without a consistency condition, the Inverse Problem of Boolean equations can be viewed as a special case of the problem of Boolean curve fitting, provided the specified points $\mathrm{z}_{k}$ are the only zeros of $f(\mathbf{X})$. Our findings are illustrated via a detailed typical example.
\end{abstract}

Keywords: Boolean curve fitting, Inverse problem, Boolean equations, Consistency condition, Uniqueness, Particular solutions.

\section{Introduction}

The problem of Boolean curve fitting (BCF), also known as Boolean interpolation, remained a pure mathematical curiosity with no view of practical applications for almost one century. Most notable among the early contributions to this problem are those due to Stamm [1], McKinsey ${ }^{[2,3]}$, Ellis ${ }^{[4,5]}$, and Scognamiglio [6]. Such contributions culminated in the classical treatise by Rudeanu ${ }^{[7]}$ in 1974. A sequel paper by Melter and Rudeanu ${ }^{[8]}$ in 1984 specialized the results in ${ }^{[7]}$ for Boolean functions that are linear in the sense of Löwenheim [9]. Boolean curve fitting witnessed a recent revival ${ }^{[10]}$, as it finally found a useful engineering application in the area of cryptography ${ }^{[11,12]}$.
The two problems are seemingly identical. So, exposing the subtle differences between them is of interest. Our comparison is a part of an ongoing effort ${ }^{[10,13]}$ to transfer these two problems from the domain of pure mathematics to the reach of engineers and problem solvers. The solutions of the two problems are being converted from declarative specifications (mathematical approach) to constructive procedures (engineering approach).

The work of Rushdi and Albarakati ${ }^{[13]}$ is related to Boolean interpolation, essentially as a special case, since it deals with the Inverse Problem of Boolean equations (IPBE), in which a Boolean function $f(\mathbf{X})$ is required to have the same value of 0 (or 1) at (and only at) 
several distinct points $\mathbf{X}=\mathbf{X}_{k}$. However, the techniques used in ${ }^{[13]}$ are not derived from or based on concepts of Boolean interpolation.

The organization of the remainder of this paper is as follows. Sections 2 and 3 review the available results on the BCF and the IPBE, respectively. Section 4 presents the mathematical relation between the BCF and the IPBE, and explains the deep similarities and subtle differences between them. Section 5 supports and clarifies the findings of Section 4 via a demonstrative example. Section 6 concludes the paper.

\section{On Boolean Curve Fitting}

In this section, we reproduce from Rudeanu ${ }^{[7]}$ and Rushdi and Balamesh ${ }^{[10]}$ the main results known on Boolean curve fitting. The problem at hand requires the determination of a Boolean curve whose graph passes through $m$ given points $\left(\mathbf{X}_{1}, z_{1}\right),\left(\mathbf{X}_{2}, z_{2}\right), \ldots,\left(\mathbf{X}_{m}, z_{m}\right)$ of the Boolean space $B^{n} \times B=B^{n+1}$, where

$\mathbf{X}_{k}=\left[X_{k, 1}, X_{k, 2}, \ldots, X_{k, n}\right]^{T} \in B^{n}, k=1,2, \ldots, m$ and $z_{k} \in B, k=1,2, \ldots, m$.

This is equivalent to finding an interpolating Boolean function $f: B^{n} \rightarrow B$ such that

$$
f\left(\mathbf{X}_{k}\right)=z_{k}, \quad k=1,2, \ldots, m
$$

The solution for the desired interpolating function $f(\mathbf{X})$ is ${ }^{[7]}$

$$
f(\mathbf{X})=\bigvee_{\mathbf{A} \in\{0,1\}^{n}}\left[\left(\bigvee_{k=1}^{m} z_{k} \mathbf{X}_{k}^{\mathbf{A}}\right) \vee p_{\mathbf{A}} \bigwedge_{k=1}^{m} \overline{\left(\mathbf{X}_{k}^{\mathbf{A}}\right)}\right] \mathbf{X}^{\mathbf{A}}
$$

where $\mathbf{A}=\left[a_{1}, a_{2}, \ldots, a_{n}\right]^{T}, p_{\mathbf{A}}$ is a parameter that belongs to the underlying Boolean algebra $B$ and $\mathbf{X}^{\mathbf{A}}$ is the primitive product (minterm) given by

$$
\mathbf{X}^{\mathbf{A}}=\mathbf{X}^{\left(a_{1}, a_{2}, \ldots, a_{n}\right)}=X_{1}^{a_{1}} X_{2}^{a_{2}} \ldots X_{2}^{a_{n}}
$$

where

$$
X_{i}^{a_{i}}=X_{i} \odot a_{i}= \begin{cases}X_{i}, & \text { if } a_{i}=1 \\ \bar{X}_{i}, & \text { if } a_{i}=0\end{cases}
$$

Equation (2) is valid subject to the consistency condition ${ }^{[7]}$

$$
\bigvee_{k=1}^{m} \bigvee_{h=1}^{m}\left[\left(z_{k} \oplus z_{h}\right) \bigwedge_{i=1}^{n}\left(X_{k, i} \odot X_{h, i}\right)\right]=0
$$

This consistency condition is needed for a solution to exist. Equation (2) reduces to the unique solution

$$
f(\mathbf{X})=\bigvee_{\mathbf{A} \in\{0,1\}^{n}}\left(\bigvee_{k=1}^{m} z_{k} \mathbf{X}_{k}^{\mathbf{A}}\right) \mathbf{X}^{\mathbf{A}}
$$

if, and only if ${ }^{[3,7]}$

$$
\bigvee_{\mathbf{A} \in\{0,1\}^{n}} \bigwedge_{k=1}^{m} \overline{\left(\mathbf{X}_{k}^{\mathbf{A}}\right)}=0
$$

\section{On the Inverse Problem of Boolean Equations}

The Forward Problem of Boolean equations (FPBE) is to find the solutions of a system of Boolean equations typically reduced to a single equation $f(\mathbf{X})=0$ (or $g(\mathbf{X})=1$ ) where $f, g: B^{n} \rightarrow B$, and $B$ is an arbitrary Boolean algebra. By contrast, the Inverse Problem of Boolean equations (IPBE) is to construct the equation $f(\mathbf{X})=0$ (or $g(\mathbf{X})=1$ ) given the consistency condition for its solution $s_{0}=0$ together with the complete set of particular solutions $\mathbf{X}_{k}, k=1,2, \ldots, m$ such that $f\left(\mathbf{X}_{k}\right)=0$ (or $g\left(\mathbf{X}_{k}\right)=1$ ) for $k=$ $1,2, \ldots, m$. Implicit in this definition is that $f(\mathbf{X}) \neq 0$ (or, respectively, $g(\mathbf{X}) \neq 1$ ) for any $\mathbf{X}$ that does not belong to the set $\left\{\mathbf{X}_{1}, \mathbf{X}_{2}, \ldots, \mathbf{X}_{m}\right\}$. Noting that $\{f(\mathbf{X})=0\}$ is implied by $\left\{s_{0}=0\right\}$ and $\left\{\mathbf{X}=\mathbf{X}_{k}, k=\right.$ $1,2, \ldots, m\}$ and nothing else, Rushdi and Albarakati ${ }^{[13]}$ obtained an expression for $f(\mathbf{X})$ that can be rewritten in the current notation as 


$$
f(\mathbf{X})=\bigvee_{\mathbf{A} \in\{0,1\}^{n}} \bigwedge_{k=1}^{m}\left(\bigvee_{i=1}^{n}\left(X_{i} \oplus X_{k, i}\right)\right) \mathbf{X}^{\mathbf{A}} \vee s_{0}
$$

Correspondingly, $g(\mathbf{X})$ is given by

$$
g(\mathbf{X})=\bigvee_{\mathbf{A} \in\{0,1\}^{n}} \bigvee_{k=1}^{m}\left(\bigwedge_{i=1}^{n}\left(X_{i} \odot X_{k, i}\right)\right) \mathbf{X}^{\mathbf{A}} \wedge \bar{s}_{0}
$$

\section{The Relation between the $\mathrm{BCF}$ and the IPBE}

The problem of Boolean curve fitting is somewhat similar to the Inverse Problem of Boolean equations when $\mathrm{z}_{k}=0$ for $k=$ $1,2, \ldots, m$. Under these conditions, the consistency condition (5) reduces to the trivial identity $\{0=0\}$, and the interpolating function (2) reduces to

$$
f_{\mathrm{BCF}}(\mathbf{X})=\bigvee_{\mathbf{A} \in\{0,1\}^{n}}\left(p_{\mathbf{A}} \bigwedge_{k=1}^{m} \overline{\left(\mathbf{X}_{k}^{\mathbf{A}}\right)}\right) \mathbf{X}^{\mathbf{A}}
$$

The complemented primtive product in (10) can be simpified via (3) and (4) to

$$
\overline{\left(\mathbf{X}_{k}^{\mathrm{A}}\right)}=\overline{\bigwedge_{i=1}^{n}\left(a_{i} \odot X_{k, i}\right)}=\bigvee_{i=1}^{n}\left(a_{i} \oplus X_{k, i}\right)
$$

Therefore, the interpolating function (10) reduces to

$$
\begin{aligned}
& f_{\mathrm{BCF}}(\mathbf{X})=\bigvee_{\mathbf{A} \in\{0,1\}^{n}} p_{\mathbf{A}} \bigwedge_{k=1}^{m}\left(\bigvee_{i=1}^{n}\left(a_{i} \oplus X_{k, i}\right)\right) \mathbf{X}^{\mathbf{A}} \\
= & \bigvee_{\mathbf{A} \in\{0,1\}^{n}} p_{\mathbf{A}} \bigwedge_{k=1}^{m}\left(\bigvee_{i=1}^{n}\left(a_{i} \oplus X_{k, i}\right)\right) \bigwedge_{i=1}^{n}\left(X_{i} \odot a_{i}\right) \\
= & \bigvee_{\mathbf{A} \in\{0,1\}^{n}} p_{\mathbf{A}} \bigwedge_{k=1}^{m}\left(\bigvee_{i=1}^{n}\left(X_{i} \oplus X_{k, i}\right)\right) \bigwedge_{i=1}^{n}\left(X_{i} \odot a_{i}\right) \\
= & \bigvee_{\mathbf{A} \in\{0,1\}^{n}} p_{\mathbf{A}} \bigwedge_{k=1}^{m}\left(\bigvee_{i=1}^{n}\left(X_{i} \oplus X_{k, i}\right)\right) \mathbf{X}^{\mathbf{A}}
\end{aligned}
$$

On the other hand, the inverse function recovered via (8), whenever no consistency condition is required $\left(s_{0}=0\right)$ is given by

$$
\begin{aligned}
f_{\mathrm{IPBE}}(\mathbf{X}) & =\bigvee_{\mathbf{A} \in\left\{0,11^{n}\right.} \bigwedge_{\substack{n=1 \\
m}}^{m}\left(\bigvee_{i=1}^{n}\left(X_{i} \oplus X_{k, i}\right)\right) \mathbf{X}^{\mathbf{A}} \\
& =\bigvee_{\mathbf{A} \in\{0,1\}^{n}} \bigwedge_{k=1}^{n}\left(\bigvee_{i=1}^{n}\left(a_{i} \oplus X_{k, i}\right)\right) \mathbf{X}^{\mathbf{A}}
\end{aligned}
$$

The function $f(\mathbf{X})$ obtained in (13) by the IPBE is exactly the same as the one obtained by the BCF in (12) except for an extra multiplicative factor $p_{\mathrm{A}}$ that appears in each cell $\mathbf{A} \in\{0,1\}^{n}$ in (12). While the function $f_{\text {IPBE }}(\mathbf{X})$ in (13) is unique, the function $f_{\mathrm{BCF}}(\mathbf{X})$ in (12) might have many values depending on the values of the parameters $p_{\mathrm{A}}$. One of the possible values of $f_{\mathrm{BCF}}(\mathbf{X})$ is $f_{\mathrm{IPBE}}(\mathbf{X})$, obtained under the conditions

$$
p_{\mathrm{A}} \bigwedge_{k=1}^{m}\left(\bigvee_{i=1}^{n}\left(a_{i} \oplus X_{k, i}\right)\right)=\bigwedge_{\substack{k=1 \\ \forall \mathbf{A} \in\{0,1\}^{n}}}^{m}\left(\bigvee_{k=1}^{n}\left(a_{i} \oplus X_{k, i}\right)\right)
$$

Or equivalently

$$
\bigwedge_{k=1}^{m}\left(\bigvee_{i=1}^{n}\left(a_{i} \oplus X_{k, i}\right)\right) \leq p_{\mathbf{A}}, \quad \forall \mathbf{A} \in\{0,1\}^{n}
$$

Conditions (14) or (15) are satisfied, in paricular, if

$$
p_{\mathbf{A}}=1, \quad \forall \mathbf{A} \in\{0,1\}^{n}
$$

Equation (16) means that a sufficient, but no necessary, condition for $f_{\mathrm{BCF}}(X)=f_{\mathrm{IPBE}}(X)$ is $p_{\mathrm{A}}=1$.

\section{Illustrative Example}

Consider the BCF problem of finding an interpolating Boolean function $f: B_{16}^{2} \rightarrow B_{16}$, where $B_{16}=\mathrm{FB}\{a, b\}$, the free Boolean algebra with generators $a$ and $b$, such that it represents a curve in the Boolean space $B_{16}^{3}$ that passes through the four points.

\begin{tabular}{|c|c|c|c|c|}
\hline$k$ & 1 & 2 & 3 & 4 \\
\hline $\mathbf{X}_{k}$ & $(a \bar{b}, \bar{a} b)$ & $(a, b)$ & $(\bar{b}, \bar{a})$ & $(a \vee \bar{b}, \bar{a} \vee b)$ \\
\hline$z_{k}$ & 0 & 0 & 0 & 0 \\
\hline
\end{tabular}


It is clear from (5) that the consistency condition for this BCF problem is the trivial identity $(0=0)$. Figures 1 and 2 demonstrate the evolution of the variable-entered Karnaugh map (VEKM) representation for $f_{\mathrm{BCF}}(\mathbf{X})$ expressed via (12). Thus, the final parametric solution can be read from the VEKM in Fig. 2 as ${ }^{[14-17]}$

$$
\begin{aligned}
f_{\mathrm{BCF}}\left(X_{1}, X_{2}\right)= & p_{0}(\bar{a} b \vee a \bar{b}) \bar{X}_{1} \bar{X}_{2} \\
& \vee p_{1}(a \vee \bar{b}) \bar{X}_{1} X_{2} \\
& \vee p_{2}(\bar{a} \vee b) X_{1} \bar{X}_{2} \\
& \vee p_{3}(a \bar{b} \vee \bar{a} b) X_{1} X_{2}
\end{aligned}
$$

where, with a little twist of notation, we are using $p_{0}, p_{1}, p_{2}$ and $p_{3}$ to stand for $p_{A} \in A=$ $\{0,1\}^{2}=\{00,01,10,11\}$ or for $p_{00}, p_{01}, p_{10}$ and $p_{11}$. The corresponding IPBE is to find the equation $f(\mathbf{X})=f\left(X_{1}, X_{2}\right)=0, \quad$ where $f: B_{16}^{2} \rightarrow B_{16}=\mathrm{FB}\{a, b\}$ such that it has a consistency condition $\{0=0\}$ and a set of particular solutions

$$
\{(a \bar{b}, \bar{a} b),(a, b),(\bar{b}, \bar{a}),(a \vee \bar{b}, \bar{a} \vee b)\}
$$

Figure 3 displays the VEKM representation for $f_{\text {IPBE }}(\mathbf{X})$ expressed via (13). Thus, the final unique solution can be read from the VEKM in Fig. 3 as ${ }^{[14-17]}$

$$
\begin{aligned}
f_{\mathrm{IPBE}}\left(X_{1}, X_{2}\right)= & (\bar{a} b \vee a \bar{b}) \bar{X}_{1} \bar{X}_{2} \\
& \vee(a \vee \bar{b}) \bar{X}_{1} X_{2} \\
& \vee(\bar{a} \vee b) X_{1} \bar{X}_{2} \\
& \vee(a \bar{b} \vee \bar{a} b) X_{1} X_{2}
\end{aligned}
$$

\begin{tabular}{|c|c|c|}
\hline & $X_{1}$ \\
\hline & $p_{0}(\bar{a} b \vee a \bar{b})$ & $p_{1}(\bar{a} \vee b)$ \\
\hline$X_{2}$ & $p_{2}(a \vee \bar{b})$ & $p_{3}(a \bar{b} \vee \bar{a} b)$ \\
\hline
\end{tabular}

Matching the terms in (17) and (19), we can see that the solution in (19) can be obtained as

\begin{tabular}{|c|c|c|}
\hline & $X_{1}$ \\
\hline & $\bar{a} b \vee a \bar{b}$ & $\bar{a} \vee b$ \\
\hline$X_{2}$ & $a \vee \bar{b}$ & $a \bar{b} \vee \bar{a} b$ \\
\hline
\end{tabular}
a special case of that in (17) under the conditions (15), namely

$$
\begin{aligned}
(\bar{a} b \vee a \bar{b}) \leq p_{0}, & (a \vee \bar{b}) \leq p_{1} \\
& (\bar{a} \vee b) \leq p_{2} \\
& (a \bar{b} \vee \bar{a} b) \leq p_{3}
\end{aligned}
$$

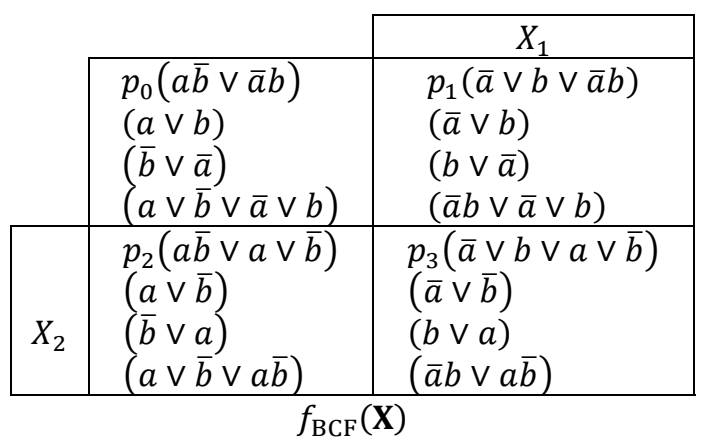

Fig. 1. Initial VKEM representation of the function obtained via the BCF.

Fig. 2. Final VKEM representation of the function obtained via the BCF.

Fig. 3. VKEM representation of the function obtained via the IPBE. 


\begin{tabular}{|c|c|c|}
\cline { 3 - 3 } \multicolumn{1}{c|}{} & $X_{1}$ \\
\cline { 2 - 3 } \multicolumn{1}{c|}{} & $C_{01} \bar{a} b \vee C_{02} a \bar{b}$ & $C_{10} \bar{a} \bar{b} \vee C_{11} \bar{a} b \vee C_{13} a b$ \\
\hline$X_{2}$ & $C_{20} \bar{a} \bar{b} \vee C_{22} a \bar{b} \vee C_{23} a b$ & $C_{31} \bar{a} b \vee C_{32} a \bar{b}$ \\
\hline \multicolumn{2}{|c}{$f_{\mathrm{BCF}}(\mathbf{X})$}
\end{tabular}

Fig. 4. VKEM representation with atomic entries of the function obtained via the BCF.

Each of the four parameters $p_{i} \in B_{16}=$ $\mathrm{FB}(a, b), 0 \leq i \leq 3$, can be expanded in terms of the four atoms of $B_{16}$ as ${ }^{[7]}$

$p_{i}=C_{i 0} \bar{a} \bar{b} \vee C_{i 1} \bar{a} b \vee C_{i 2} a \bar{b} \vee C_{i 3} a b$,

$C_{i j} \in\{0,1\}, 0 \leq i \leq 3$

Note that the expansions in (21) involve $4 \times 4=16$ binary coefficients. The entries of the VEKM in Fig. 2 can now be expressed as

$$
\begin{aligned}
& p_{0}(a \bar{b} \vee \bar{a} b)=C_{01} \bar{a} b \vee C_{02} a \bar{b} \\
& p_{1}(\bar{a} \vee b)=C_{10} \bar{a} \bar{b} \vee C_{11} \bar{a} b \vee C_{13} a b \\
& p_{2}(a \vee \bar{b})=C_{20} \bar{a} \bar{b} \vee C_{22} a \bar{b} \vee C_{23} a b \\
& p_{3}(a \bar{b} \vee \bar{a} b)=C_{31} \bar{a} b \vee C_{32} a \bar{b}
\end{aligned}
$$

We now replace the VEKM of Fig. 2 by the one of atomic entries in Fig. 4, in which 10 only of the aforementioned binary coefficients remain. Since each of these coefficients can be assigned two values independently of the others, the number of distinct particular solutions expressed by $f_{\mathrm{BCF}}(\mathbf{X})$ in (17) is $2^{10}=1024$. This is the number of curves passing through the four points in the given Boolean space.

\section{Conclusion}

This paper explores the relation between the problem of Boolean curve fitting (BCF) and the problem of the Inverse Problem (IPBE) of Boolean equations. Despite the great similarity between the two problems, there is a subtle difference between them as the first might require a consistency condition for its solution while the second might use such a condition as input. Without a consistency condition in either problem, the two problems yield solutions that differ only via an arbitrary multiplicative parameter needed in the $\mathrm{BCF}$ problem. The $\mathrm{BCF}$ problem might have conditioned or unconditioned solutions that might be numerous or unique or it might have no solutions at all. By contrast, the IPBE is guaranteed to have a unique solution that happens to be one of those produced by the BCF problem.

\section{References}

[1] Stamm, E., Geometrische Theorie logischer Funktionen: Beitrag zur Algebra der Logik (Geometrical theory of logical functions: Contribution to the algebra of logic), Prace MatematycznoFizyczne, 34(1): 119-158 (1925), In German.

[2] McKinsey, J. C. C., On Boolean functions of many variables, Transactions of the American Mathematical Society, 40(3): 343-362 (1936).

[3] McKinsey, J. C. C., Boolean functions and points, Duke Mathematical Journal, 2(3): 465-471 (1936).

[4] Ellis, D., Remarks on Boolean functions, Journal of the Mathematical Society of Japan, 5(3-4): 345-350 (1953).

[5] Ellis, D. Remarks on Boolean functions II, Journal of the Mathematical Society of Japan, 8(4): 363-368 (1956).

[6] Scognamiglio, G., Interpolazione per le funzioni algebriche booleane (Interpolation for Boolean algebraic functions), Giorn. Mat. Battaglini, 89: 14-41 (1961), In Italian.

[7] Rudeanu, S., Boolean Functions and Equations, North-Holland Publishing Company \& American Elsevier, Amsterdam, the Netherlands (1974).

[8] Melter, R. A. and Rudeanu, S., Linear equations and interpolation in Boolean algebra, Linear Algebra and Its Applications, 57: 31-40 (1984).

[9] Löwenheim, L., Gebietsdeterminanten (Domain determinants), Mathematische Annalen, 79(3): 223 236 (1918), In German.

[10] Rushdi, A. M. and Balamesh, A. S., Boolean Curve 
Fitting with the Aid of the Variable-Entered Karnaugh Map, forthcoming ISA.

[11] Ahmad, W. and Rushdi, A. M. A., A new cryptographic scheme utilizing the difficulty of big Boolean satisfiability, International Journal of Mathematical, Engineering and Management Sciences (IJMEMS), 3(1): 47-61 (2018).

[12] Balamesh, A. S., and Rushdi, A. M., Implementation and cryptanalysis of a novel cryptosystem based on Boolean curve fitting over a big Boolean algebra, forthcoming ISA.

[13] Rushdi, A. M. A. and Albarakati, H. M., The inverse problem for Boolean equations, Journal of Computer Science, 8(12): 2098-2105 (2012).

[14] Rushdi, A. M., Improved variable-entered Karnaugh map procedures, Computers and Electrical Engineering, 13(1): 41-52 (1987).

[15] Rushdi, A. M. and Al-Yahya, H. A., A Boolean minimization procedure using the variable entered
Karnaugh map and the generalized consensus concept, International Journal of Electronics, 87(7): 769-794 (2000).

[16] Rushdi, A. M. and Al-Yahya, H. A., Further improved variable-entered Karnaugh map procedures or obtaining the irredundant forms of an incompletelyspecified switching function, Journal of King Abdulaziz University: Engineering Sciences, 13(1): 111-152 (2001).

[17] Rushdi, A. M., and Amashah, M. H., Using variable-entered Karnaugh maps to produce compact parametric general solutions of Boolean equations, International Journal of Computer Mathematics, 88(15), 3136-3149 (2011). 


\section{حول العلاقة بين مواعمة المنحنيات البولانية والمسألة العكسية للمعادلات البولانية}

\section{علي محمد رشدي و أحمد سعيد بالعمش}

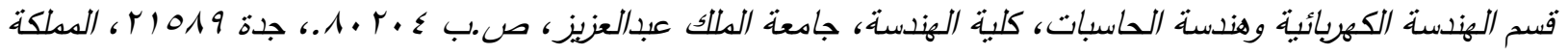
العربية السعودية

arushdi@kau.edu.sa

الدستخلص. تستكثف ورقة البحث هذه الثثابهات والاختلافات بين مسألتين بارزتين في

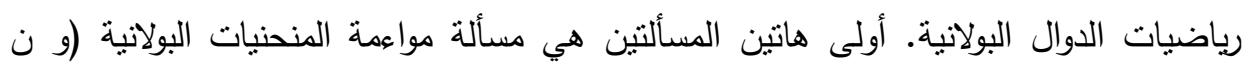

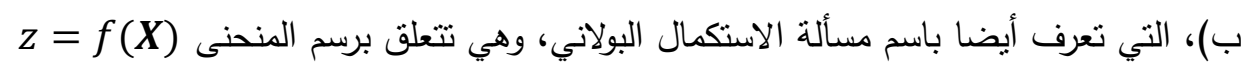

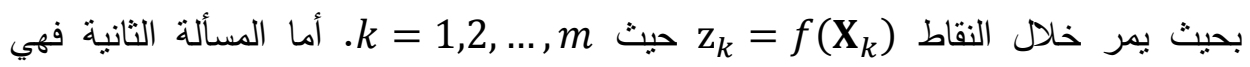
المسألة العكسية (س ع) للمعادلات البولانية التي توجد دالة بولانية (X)

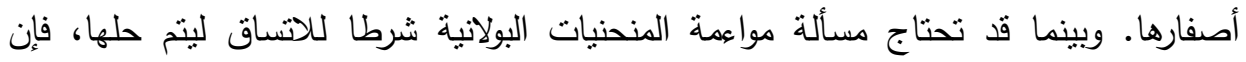
المسألة العكسية للمعادلات البولانية ربما نستخدم شرط اتساق كمدل لهان لها. وفي حالة انعدام

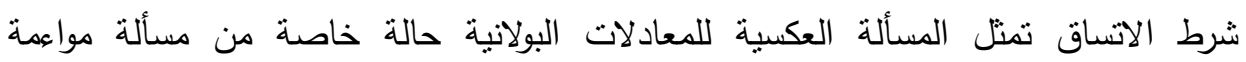
المنحنيات البولاثية، شريطة أن تكون قيم النقاط المحددة

$$
\text { توضيح نتائجنا من خلال مثال تقصيلي نمطي. }
$$

كلمات مفتاحية: مواءمة المنحنيات البولانية، المسألة العكسية، المعادلات البولانية، شرط

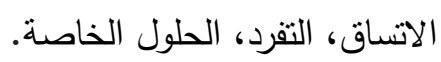


